\title{
Benign paroxysmal positional vertigo of the horizontal canal
}

\author{
G De la Meilleure, I Dehaene, M Depondt, W Damman, L Crevits, G Vanhooren
}

\begin{abstract}
Objectives-To review the clinical features, electronystagmography findings, the possible mechanism, and a possible therapeutic approach to benign paroxysmal positional vertigo (BPPV).

Methods-Sixty three cases of BPPV of the horizontal canal type have been reviewed. It is characterised by horizontal nystagmus and an intense vertigo, provoked by rotation of the head in a supine patient. The horizontal nystagmus beats towards the ground on both sides, becomes more pronounced when lying on the pathological side, and then the nystagmus often changes direction.

Results-Forty eight patients underwent electronystagmography. On the pathological side, the first phase nystagmus had a mean latency of three seconds and a mean duration of 31.6 seconds. Nystagmus inversion occurred in 36 patients after a nystagmus free interval. The mean second phase nystagmus duration lasted 33.4 seconds. On the healthy side, the nystagmus had a mean latency of 3.4 seconds and a mean duration of 39.5 seconds. Fatigue was seen in six patients. Simultaneous involvement of the posterior canal was present in 16 patients. A liberatory manoeuvre was successful in six patients.

Conclusions-The liberatory manoeuvre should be tried in patients with horizontal canal vertigo. It should not be performed in patients with severe cervical arthrosis, vertebrobasilar insufficiency, or when the patient has neck pain during the manoeuvre.
\end{abstract}

\section{$(尹$ Neurol Neurosurg Psychiatry 1996;60:68-71)}

Keywords: horizontal benign positional vertigo; nystagmography

Benign paroxysmal positional vertigo (BPPV) is usually due to involvement of the posterior semicircular canal. The diagnosis is confirmed by provoking the vertigo and the typical verticorotatory nystagmus with the Dix-Hallpike manoeuvre. ${ }^{1}$ The nystagmus appears with a latency of 1 to 15 seconds. ${ }^{2}$ Its duration is usually less than 30 seconds. Fatigue with repeated positioning is seen. ${ }^{34}$ The nystagmus characteristics can be attributed to an ampullofugal displacement of the ampulla of the posterior semicircular canal. ${ }^{56}$
The horizontal canal variant of BPPV is less frequent. $\mathrm{McClure}^{7}$ reported seven cases, characterised by intense vertigo and pure horizontal nystagmus. Pagnini et $a l^{8}$ published 15 cases of horizontal canal BPPV. Recently Baloh $e t a l^{9}$ added another 13 cases.

The vertigo in horizontal canal BPPV can be provoked by turning the head to either side in the supine position. It is always more pronounced on the pathological side. The patient can get up or lie down, bend or straighten up with only minimal complaints. The vertigo is characterised by a short latency, a very sudden onset, and a duration longer than 30 seconds. It is more intense than in the posterior canal variant and is usually associated with severe autonomic symptoms. ${ }^{8-10}$

On the pathological side a more intense, paroxysmal, geotropic (beating toward the ground), and purely horizontal nystagmus is seen after a short latency period. Often a nystagmus inversion without changing head position is seen. This nystagmus is apogeotropic (beating away from the ground) and less paroxysmal with a smaller amplitude and frequency. When rolling the head to the healthy contralateral side, a less intense horizontal geotropic paroxysmal nystagmus is seen after a short latency period. ${ }^{8 \cdot 10}$

When a verticorotatory nystagmus is also present, it indicates a combination of posterior and horizontal BPPV. ${ }^{89}$ We report 47 cases of pure horizontal canal BPPV and 16 cases of combined posterior and horizontal canal BPPV. In 48 patients electronystagmography (ENG) was available. These data enable us to discuss the clinical features, the ENG findings, the hypothetical mechanism, and a possible therapeutic approach.

\section{Methods}

We reviewed retrospectively the clinical records of 287 patients with BPPV over a four year period. All patients were systematically tested for horizontal and posterior canal involvement. A total of 63 patients $(22 \%)$ showed horizontal canal involvement, of whom $16(5 \%)$ had posterior canal involvement and $47(16 \%)$ did not. There were 28 men and 35 women and they had an overall age distribution with a unimodal normal aspect (mean (SD) age 58.1 (12.9), range 27-86 years).

All the patients had clinical features compatible with horizontal canal BPPV. Diagnosis was made by provoking the typical nystagmus by a rapid lateral rotation of the head to either 


\begin{tabular}{lc} 
Clinical features & \\
\hline No of patients & 63 \\
Age (range) (y) & $27-86$ \\
(Mean (SD)) & $58 \cdot 1(12 \cdot 9)$ \\
Single vertiginous episode & 38 \\
Multiple vertiginous episodes & 25 \\
Lateralisation horizontal canal involvement: & \\
Right & 33 \\
Left & 30 \\
Concommitant posterior canal involvement & 16 \\
Bilateral & 2 \\
Unilateral & 14 \\
$\quad$ Ipsilateral & 10 \\
$\quad$ Contralateral & 4 \\
Vertigo severity scale: & \\
1 & 9 \\
2 & 31 \\
3 & 23 \\
Hearing impairment: & \\
Bilateral & 11 \\
Unilateral & 9 \\
$\quad$ Ipsilateral & 4 \\
Contralateral & 5 \\
Tinnitus: & \\
Bilateral & 4 \\
Unilateral & 10 \\
Ipsilateral & 5 \\
Contralateral & 5 \\
Vestibular hyporeflexia: & \\
Bilateral & 3 \\
Unilateral & 8 \\
Ipsilateral & 4 \\
$\quad$ Contralateral & 4 \\
\hline &
\end{tabular}

side through 90 degrees with the patient lying supine. Each patient was also tested for posterior canal involvement by performing the DixHallpike manoeuvre bilaterally. ${ }^{1}$ For the evaluation of the severity of the vertiginous complaints from the clinical records, we developed the following scale: slight vertigo in the provoking position without autonomous symptoms scored 1; severe vertigo in the provoking position with nausea scored 2 and severe vertigo in the provoking position with severe nausea, vomiting, or hypotension scored 3.

In 48 patients ENG was performed, which documented the nystagmus. Repeated testing (at least twice with short intervals) during the registration enabled us to obtain information about the tendency to fatigue. Twenty three right sided and 25 left sided cases of horizontal canal BPPV were evaluated.

Hearing impairment was defined as more than $15 \mathrm{~dB}$ average hearing loss in the frequency range 250 to $4000 \mathrm{~Hz}$ and vestibular hyporeflexia was defined as pathological when the calculated vestibular paresis was larger than $22 \%{ }^{11}$

No specific treatment was prescribed in 57 patients. In six patients a liberatory manoeuvre was performed. The patient was placed in the supine position on an examining table. The head was turned to either side to provoke the horizontal canal nystagmus. The pathological

Figure 1 Distribution of symptom duration in patients with single and multiple episode horizontal canal BPPV. Twenty seven patients experienced only a single episode with a duration less than one month. Sixteen patients experienced multiple episodes with a duration of more than one year.

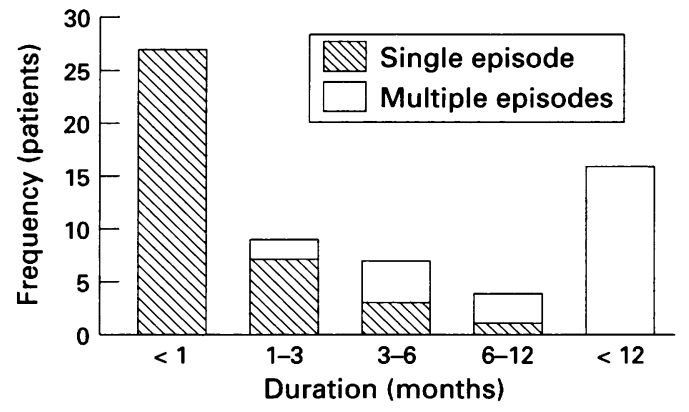

side was determined by evaluating the intensity of the vertigo and nystagmus. In the neutral supine position, the head was lifted 30 degrees away from the table to bring the horizontal canal in a plane of maximal influence, and then turned to the pathological side. The typical geotropic nystagmus for the pathological side was provoked. The head stayed in this lateral position for five minutes. Then the head was turned as fast as possible to the other side over 180 degrees, keeping the head 30 degrees flexed. The typical less intense geotropic nystagmus for the healthy side was provoked. Again the head stayed in this lateral position for five minutes. Afterwards the patient was asked to avoid lying down for the next 48 hours and to avoid head shaking.

\section{Results}

The table presents the clinical features. The aetiologically important factors were idiopathic (40 patients), traumatic (15), general surgery (three), stapedectomy (one), viral infection (two), and bed rest (two).

Thirty eight patients experienced only a single episode of horizontal canal BPPV with a symptom duration varying from less than one month to one year. However, 25 patients had multiple episodes of vertigo with a total duration varying from one month to several years (fig 1). Silent periods existed between the episodes.

On ENG recordings (fig 2), latency varied on both sides between one and eight seconds. The first phase nystagmus duration at the pathological side varied between 14 and 71 seconds. Nystagmus inversion was seen on the pathological side in 36 patients and it never occurred on the healthy side. The duration of a nystagmus free interval varied between 3 and 22 seconds. The inverse second phase nystagmus lasted between 5 and 62 seconds. The duration of the nystagmus at the healthy side lasted between 13 and 97 seconds. Recording confirmed fatigue in six patients.

Two patients with a pure horizontal canal BPPV were treated with the liberatory manoeuvre. The vertigo was present for one week in one and for one month in the other patient. Both still had severe vertiginous complaints on the day the manoeuvre was performed. The next day the vertigo and nystagmus could no longer be provoked. Three patients had a combined posterior and horizontal canal BPPV. The vertigo was present between two weeks and two months. In all three patients the horizontal canal component was treated first. When the patient was re-evaluated three days later, the horizontal canal component had disappeared. The posterior canal was treated successfully with one Sémont manoeuvre in each case. ${ }^{12}$ These five patients had no recurrences in the follow up period of more than one year.

Finally, one patient had bilateral posterior canal involvement and right horizontal canal involvement. We performed the manoeuvre for the right side. Three days later the left side had the typical signs of horizontal canal 
A

Pathological side

$\mathrm{H}$ C

Non-pathological side

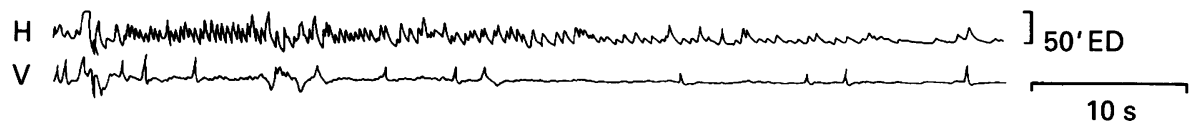

B

Pathological side

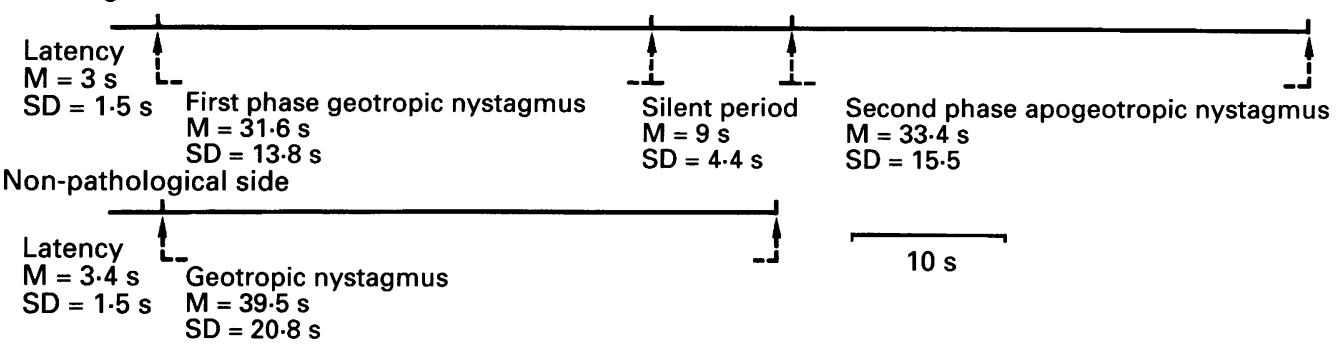

Figure 2(A) ENG of a left sided horizontal canal BPPV, showing the typical nystagmus, for both the pathological and the non-pathological side (ED = eye deviation). Upward deflections indicate rightwards eye movement in the horizontal $(H)$ trace and upward eye movements in the vertical $(V)$ trace. (B) Graphical representation of the mean (M) and standard deviation (SD) of the latencies, the duration of the silent period, and the nystagmus phases for both pathological and non-pathological side. $M$ and SD were based on the results of the ENG recordings in 48 patients, except for the values of the second phase apogeotropic nystagmus, which were based on the results of the 36 patients with nystagmus inversion.

involvement. The patient was now treated for the left side, which eliminated the horizontal component. Two Sémont liberatory manoeuvres for each side were necessary to cure the posterior canal component. One month later a recurrence of the left posterior canal component occurred. The follow up period is now one year, without recurrences.

\section{Discussion}

Horizontal canal BPPV is more frequent than previously mentioned, ${ }^{8-10}$ as it comprised $22 \%$ of all our patients with BPPV. The occurrence of horizontal canal BPPV is more or less equally divided between men and women. The mean age of 58.1 years for the first episode indicates that patients over 40 are more prone to develop the disease, which is compatible with the proposed theory of utricular degeneration. ${ }^{1314}$ Most cases are idiopathic, followed by those of traumatic origin. The distribution of the aetiological factors is the same as in posterior canal BPPV, ${ }^{315}$ suggesting a similar pathophysiological mechanism. None of the patients showed clinical or ENG signs of CNS pathology.

The canalolithiasis theory explains the features of posterior canal BPPV. ${ }^{16-18}$ The degenerative debris are free floating in the endolymph and not adherent to the cupula (cupulolithiasis). ${ }^{1314}$ The particles of debris, detached from the otoconial layer by degeneration or head trauma, gravitate into the posterior canal, where they form a plug floating in its ampullofugal branch. ${ }^{17} 18$ In the provoking head-hanging Dix-Hallpike position the endolymph pulls on the cupula, because the free floating otoconia fall under the influence of gravity. ${ }^{16}$ Nystagmus latency, usually between five and 15 seconds, is explained by inertia of the clot. ${ }^{9}$ The cupula deflection ends when the clot reaches its lowest position and accounts for the limited duration of the nystagmus. ${ }^{16}$ Fatigue is due to dispersion of the clot particles and reactivation after bedrest is caused by renewed clot formation. Liberatory manoeuvres are effective in sluicing down the particles through the upper ampullofugal branch. ${ }^{18}$

The canalolithiasis theory can also explain the horizontal canal variant (fig 3). ${ }^{7917}$ Degenerative debris enter the nonampullary side of the pathological horizontal canal when the patient lies supine. When rotating the head rapidly to the pathological side, gravity and the angular head acceleration make the mass descend in the canal toward the ampulla. The movement of the clot continues until the deepest position is reached and provokes an ampullopetal deviation of the cupula. A burst of geotropic nystagmus is seen. When maintaining the head rotation to the pathological side, a burst of apogeotropic nystagmus can be seen, due to an inversion of the direction of clot movement. This can also be obtained by returning the head to the original position. When the head is rotated to the healthy side the mass is displaced further toward the ampullofugal end of the canal with an ampullofugal displacement of the cupula and a geotropic nystagmus occurs. Latency is usually shorter in horizontal canal BPPV, but sometimes it is as long as eight seconds. Baloh et al suggested that the nature of the debris could be different in posterior and horizontal canal BPPV. ${ }^{9}$ The longer latencies noted in our series suggest that the pathophysiological mechanism can be explained by calcium carbonate crystals. By contrast with previous reports, ${ }^{89}$ fatigue was seen in six patients and it can be explained by dispersion of the crystals.

The combination of posterior and horizontal BPPV in the same patient ${ }^{810}$ supports the canalolithiasis theory, as the ampullary aperture of the posterior canal and the non- 
Figure 3

Pathophysiological

mechanism in right sided horizontal canal

$B P P V$. (A) In the neutral supine position the debris clot is located at the lowest part of the horizontal canal. When the head is turned to the right side, the clot moves toward the ampulla $(A)$ and an ampullopetal displacement of the cupula occurs $\left(A^{\prime}\right)$, giving rise to the geotropic nystagmus, often followed by an apogeotropic nystagmus when the clot starts to fall back, displacing the cupula in an ampullofugal direction.

(B) When the head is turned to the left, the clot becomes displaced toward the non-ampullary aperture $(B)$ and the cupula moves ampullofugally $\left(B^{\prime}\right)$, giving rise to a geotropic

nystagmus. (C) Treatment by a liberatory manoeuvre: the head is quickly turned from the right lateral position to the left lateral position, keeping the head 30 degrees flexed. The clot is forced through the nonampullary aperture (C). Again the cupula is displaced ampullofugally $\left(C^{\prime}\right)$ and a geotropic nystagmus is provoked.
A
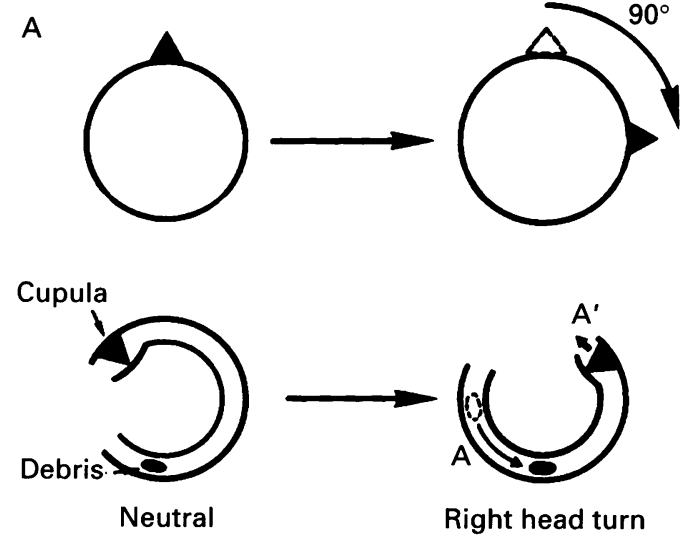

B

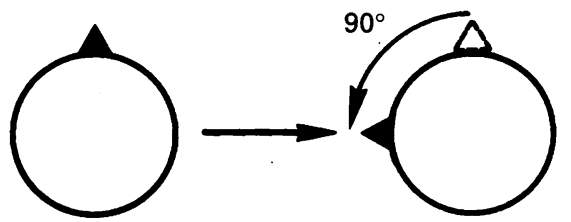

Cupula

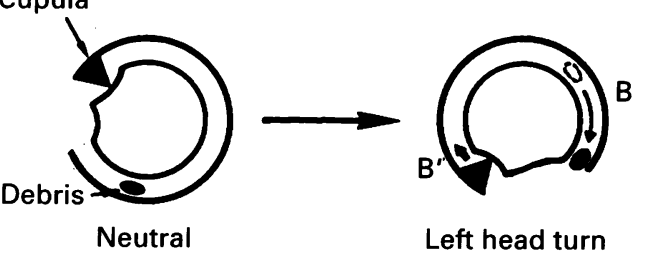

C

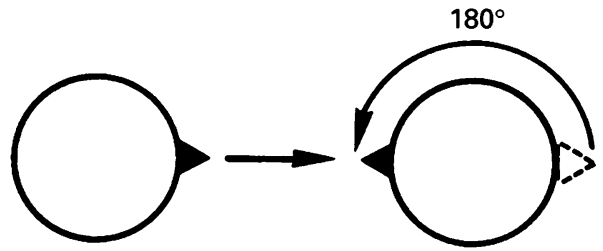

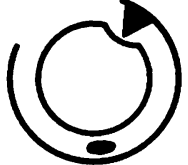

Right head turn

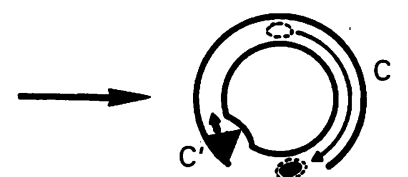

Left head turn ampullary aperture of the horizontal canal are the undermost in a standing patient. The inorganic particles tend to fall into those apertures, with a predilection for the posterior canal. Most of our patients with the combined form had ipsilateral involvement of the horizontal and posterior canal. This contrasts with the data of Baloh et al, ${ }^{9}$ who found only crossed involvement in his three patients

Hearing impairment was present in about one third of our patients. It is not helpful in determining the pathological side, as it is often bilateral. When unilateral, it is about equally divided between the pathological and the nonpathological side. The same is true for tinnitus and vestibular hyporeflexia.

Contrary to posterior canal BPPV, ENG recordings can be easily obtained in the horizontal canal form. Our findings confirm the previously described features of nystagmus. ${ }^{8-10}$ The nystagmus inversion on the pathological side was seen in $75 \%$ of the ENG registrations. Inversion was not seen on the healthy side. The reversal of nystagmus can be explained by adaptation ${ }^{9}$ or by reversal of the clot movement direction due to gravitational forces.

Recently Baloh et $a l^{9}$ concluded that there is no good therapeutic exercise or liberatory manoeuvre available. Lempert ${ }^{19}$ reported two patients treated by a liberatory manoeuvre with head rotation over 270 degrees. Our liberatory manoeuvre is also effective, as we obtained good results in all six patients treated. On rolling the head toward the pathological side (fig 3), the debris sink ampullopetally in the horizontal canal and cause the typical geotropic nystagmus. When the head is turned quickly to the other side over 180 degrees, the debris will be forced through the non-ampullary aperture into the utriculus if the velocity of the movement is large enough. However, further confirmation of this treatment on a larger group of patients with longer follow up is necessary.

We propose that this therapeutic approach should be considered for all patients with horizontal canal vertigo, as this disease can be very disabling for the patient. This manoeuvre should not be performed in patients with severe cervical arthrosis, vertebrobasilar insufficiency, or when the patient complains of neck pain while performing the provoking manoeuvre.

1 Dix MR, Hallpike CS. Pathology, symptomatology and diagnosis of certain common disorders of the vestibular system. Ann Otol Rhinol Laryngol 1952;6:987-1016.

2 Bourgeois PM, Dehaene I. Benign paroxysmal positional vertigo (BPPV): clinical features in 34 cases and review of literature. Acta Neurol Belg 1988;88:65-74.

3 Baloh RW, Honrubia V, Jacobson K. Benign positonal vertigo: clinical and oculographic features in 240 cases. Neurology 1987:37:371-8.

4 Katzarkas A. Nystagmus of paroxysmal positional vertigo: some new insights. Ann Otol Rhinol Laryngol 1987;96: 305-8.

5 Cohen B, Tokumasu K, Goto K. Semicircular canal nerve, eye and head movements: the effect of changes in initial eye and head position on the plane of the induced movement. Arch Ophthalmol 1966;76:523-31.

6 Gacek RR. Further observations on posterior ampullary nerve transection for positional vertigo. Ann Otol Rhinol Laryngol 1978;87:300-5.

7 McClure JA. Horizontal canal BPV. Am $f$ Otolaryngol 1985;4:30-5

8 Pagnini P, Nuti D, Vannucchi P. Benign paroxysmal vertigo of the horizontal canal ORL $\mathcal{F}$ Otorhinolaryngol Relat Spec 1989;51:161-70.

9 Baloh RW, Jacobson K, Honrubia V. Horizontal semicircular canal variant of benign positional vertigo. Neurology 1993:43:2542-9.

10 McClure JA. Functional basis for horizontal canal BPV. In: Barber HO, Scharpe JO, eds. Vestibular disorders Chicago: Yearbook Medical Publishers 1988:233-8.

11 Baloh RW, Honrubia V. Clinical neurophysiology of the vestibular system. Philadelphia: FA Davis Co, 1979:140.

12 Sémont A, Freyss G, Vitte E. Curing the BPPV with a liberatory maneuver. Adv Otorhinolaryngol 1988;42:290-3.

13 Schuknecht H. Cupulolithiasis. Arch Otolaryngol Head neck Surg 1969:765-8.

14 Schuknecht H, Ruby R. Cupulolithiasis. Adv Otorhinolaryngol 1973;20:434-3.

15 Brandt Th. Vertigo: its multisensory syndromes. Benign paroxysmal positioning vertigo (BPPV). London: SpringerVerlag, 1991:139-51.

16 Hall SF, Ruby RRF, McClure JA. The mechanisms of benign paroxysmal vertigo. f Otolaryngol 1979;8:151-8.

17 Brandt Th, Steddin S. Reply to the letter by Gordon. "Benign presm l. Reply to the letter by Gordon: "Benign paroxysmal positional vertigo (BPPV) or bubble

18 Brandt paroxysmal positioning vertigo, revisited. Neurology paroxysmal posi 19 Lempert Th: Horizontal benign positional vertigo [letter] 